PROCEEDINGS OF THE

AMERICAN MATHEMATICAL SOCIETY

Volume 133, Number 5, Pages 1317-1323

S 0002-9939(04)07689-0

Article electronically published on November 22, 2004

\title{
DEGENERATIONS OF CUBIC THREEFOLDS AND MATROIDS
}

\author{
TAWANDA GWENA
}

(Communicated by Michael Stillman)

\begin{abstract}
We present a surprising connection between cubic threefolds and the well-known regular matroid $R_{10}$ by making use of intermediate Jacobians of cubic threefolds realized as Prym varieties. As a corollary we obtain a new proof of the nonrationality of generic cubic threefolds.
\end{abstract}

\section{INTRODUCTION}

This paper uses the methods of degenerations of Prym varieties of $\mathrm{ABH}$ to establish a surprising new connection between cubic threefolds and a very wellknown regular matroid called $R_{10}$. As in $[\mathrm{ABH}]$ we will work over an arbitrary algebraically closed field of characteristic not 2 .

Recall that the Jacobian $J C$ of a smooth curve $C$ is an algebraic variety parametrizing line bundles on $C$ of degree 0 . Let $\mathcal{R}_{g}$ be the moduli space of smooth curves of genus $2 g-1$ with involution $\iota$, and let $\overline{\mathcal{R}}_{g}$ be its compactification obtained by adding stable curves. Also, let $\mathcal{A}_{g}$ be the moduli space of principally polarized Abelian varieties of dimension $g$. The Prym map $P: \mathcal{R}_{g} \rightarrow \mathcal{A}_{g-1}$ is given by setting $P(\tilde{C}, \iota)$ to be the identity component of the kernel of the norm map $J \tilde{C}$ to $J(\tilde{C} / \iota)$. The Prym map extends to a rational map from $\overline{\mathcal{R}}_{g}$, the space of admissible double covers of curves, to $\overline{\mathcal{A}_{g-1}^{\text {vor }}}$, the toroidal compactification of $\mathcal{A}_{g-1}$ for the second Voronoi fan. Where this map is regular is given by $[\mathrm{ABH}]$, Theorem 3.2. We will adapt this result in Lemma 4.

A system of $m \geq n$ vectors $R$ generating $\mathbb{R}^{n}$ is called a unimodular system or regular matroid if, when we write any $n$ vectors of $R$ as columns in terms of a basis $B \subset R$ we obtain a matrix that is totally unimodular, i.e., the maximal minors are either 0,1 or -1 . Such a unimodular system of vectors can be represented by a matrix $\left(I_{n} \mid A\right)$ where the columns are the vectors. From the unimodular system of vectors above we can obtain a lattice dicing. The vectors define a family $H(R)$ of parallel hyperplanes $H(\mathbf{r}, z)=\left\{\mathbf{x} \in \mathbb{R}^{n}: \mathbf{x} \cdot \mathbf{r}=z\right\}, z \in \mathbb{Z}, \mathbf{r} \in R$. If $B \subset R$ is a basis for $\mathbb{R}^{n}$, then the intersection points of hyperplanes in $H(B)$ is a lattice $L$ and $H(R)$ is then called a lattice dicing. The set of intersection points of hyperplanes of $H(R)$ is $L$ if and only if $R$ is a unimodular system of vectors. There are three basic types of unimodular systems of vectors: cographic, graphic, and the special one called $R_{10}$, which is neither graphic nor cographic ( $\mathrm{O}$, Cor. 13.2.5). A celebrated theorem of Seymour on matroid decompositions shows that all unimodular systems

Received by the editors April 17, 2003 and, in revised form, January 29, 2004.

2000 Mathematics Subject Classification. Primary 14H40; Secondary 05B35, 14E08, 14D20.

(C)2004 American Mathematical Society

Reverts to public domain 28 years from publication 
of vectors can be obtained from amalgams of the three types mentioned above ([DG], [, 13.2.4]).

Degenerating families of Jacobians of curves give rise to cographic unimodular systems of vectors (described in Section 1). Degenerating families of Prym varieties also give rise to cell decompositions, and under special situations, as in the next section, we do get a lattice dicing, hence a unimodular system of vectors. The question that arises is: Which unimodular systems of vectors do degenerations of Prym varieties give? In this paper we obtain $R_{10}$ from degenerations of Prym varieties.

Recall that the well-known Segre Cubic is a singular cubic threefold with ten simple nodes as singularities. Mumford's construction ([CG], Appendix C) associates to a cubic threefold an intermediate Jacobian realized as a Prym variety. A family of cubic threefolds degenerating to the Segre Cubic gives a degenerating family of Prym varieties. Our main result is

Theorem 1. The unimodular system of vectors associated to this family of Prym varieties is $R_{10}$.

As a corollary of Theorem 1 we will easily obtain the following result:

Theorem 2 ([C]). Fix an algebraically closed field $k$ of characteristic not two. Then a generic cubic threefold over $k$ is not birational to $\mathbb{P}^{3}$.

In section 4 we will give the proof and a precise meaning of generic. This theorem was proved in [C] by a different degeneration method. In fact, Clemens and Griffiths ([CG]) showed that over $\mathbb{C}$ every smooth cubic threefold is not rational, and Murre extended the result to any algebraically closed field of characteristic not two ([M2]).

\section{Degenerations of Prym varieties}

We first review degenerations of Jacobians of curves in order to introduce the different types of unimodular systems of vectors. Suppose we have a degenerating family of Jacobians of curves of genus $g$ with central fiber $C$, a stable curve. This degenerating family gives a cell decomposition of a real vector space ([ABH], Section 3.1). This decomposition depends only on $C$. Let $\Gamma=\Gamma(C)$ be the dual graph of $C$. The vertices $\left\{v_{i}\right\}_{i \in I}$ correspond to irreducible components of $C$ and the edges $\left\{e_{j}\right\}_{j \in J}$ correspond to the nodes. We have a chain complex $C_{\bullet}$ with

$$
C_{0}(\Gamma, \mathbb{Z})=\bigoplus_{i \in I} v_{i} \mathbb{Z}, \quad C_{1}(\Gamma, \mathbb{Z})=\bigoplus_{j \in J} e_{j} \mathbb{Z} .
$$

Let $H_{1}(\Gamma, \mathbb{Z})$ be the first homology group. There is an embedding $H_{1}(\Gamma, \mathbb{Z}) \hookrightarrow$ $C_{1}(\Gamma, \mathbb{Z})$. The cell decomposition obtained by intersecting the subspace $H_{1}(\Gamma, \mathbb{R}) \subset$ $C_{1}(\Gamma, \mathbb{R})$ with the standard cubes in $C_{1}(\Gamma, \mathbb{R})$ is called a cographic dicing and the associated unimodular system is called cographic. The cell decomposition associated to the degeneration of curves with central fiber $C$ is this cographic dicing defined by $C$. Conversely, given a cographic unimodular system we can construct a degenerating family of Jacobians of curves that have that unimodular system. If a cographic unimodular system of vectors with $m \geq n$ vectors in $\mathbb{R}^{n}$ is $\left(I_{n} \mid A\right)$, then $\left(-A^{T} \mid I_{m-n}\right)$ is also a unimodular system of vectors. These are the graphic unimodular systems of vectors.

We now review degenerations of Prym varieties from $[\mathrm{ABH}]$, Section 3. A degenerating family of Prym varieties produces a cell decomposition of a real vector 
space ( $[\overline{\mathrm{ABH}}], 3.2(\mathrm{PP} 6))$. If the map is regular at the central fiber, then we obtain the simplest type of cell decomposition, which is a lattice dicing. We construct it as follows.

Definition 3. Let the dual graph of $\tilde{C}$ (a curve with involution $\iota$ ) be $\tilde{\Gamma}$. We obtain a cell decomposition by the following: We define a map

$$
\begin{aligned}
\pi^{-}: H_{1}(\tilde{\Gamma}, \mathbb{Z}) & \longrightarrow H_{1}\left(\tilde{\Gamma}, \frac{1}{2} \mathbb{Z}\right) \\
h & \longmapsto \frac{1}{2}(h-\iota(h)) .
\end{aligned}
$$

Let $X^{-}:=\pi^{-}\left(H_{1}(\tilde{\Gamma}, \mathbb{Z})\right)$. The space $X^{-} \otimes \mathbb{R}$ is contained in $C_{1}(\tilde{\Gamma}, \mathbb{R})$. Each edge $e_{j}$ of $\tilde{\Gamma}$ defines a coordinate function $z_{j}$ in $C_{1}(\tilde{\Gamma}, \mathbb{R})$. Let $m_{j}=1$ if $z_{j}: X^{-} \rightarrow \mathbb{Z}$ is surjective and $m_{j}=2$ if $z_{j}: X^{-} \rightarrow \frac{1}{2} \mathbb{Z}$ is surjective. The functions $m_{j} z_{j}$ define a cell decomposition of $X^{-} \otimes \mathbb{R}$.

We adapt the main results of $[\mathrm{ABH}]$ and $[\mathrm{V}]$ to give a condition for where the map $\overline{\mathcal{R}}_{g} \rightarrow \overline{\mathcal{A}}_{g-1}^{\text {vor }}$ is regular.

Lemma 4 ( $[\mathrm{V}]$, Theorem 0.1, $[\mathrm{ABH}]$, Theorem 3.2). Let $(\tilde{C}, \iota)$ be given. If there do not exist two connected subgraphs $\Gamma_{0}, \Gamma_{1}$ of the dual graph of $\tilde{C}$ such that $\iota\left(\Gamma_{i}\right)=\Gamma_{i}(i=0,1)$, connected by $2 n \geq 4$ interchanged edges, then the rational map $\overline{\mathcal{R}}_{6} \rightarrow \overline{\mathcal{A}_{5}^{\text {vor }}}$ is regular in the neighborhood of $(\tilde{C}, \iota)$ and for the image point, the cell decomposition defined in ( $(\mathrm{ABH}]$, 3.2, (PP6)) equals the decomposition defined above.

\section{PRYM VARIETIES OF CUBiC THREEFolds}

The Prym variety associated to a smooth cubic threefold $X$ in $\mathbb{P}^{4}$ is constructed as follows. The lines in $X$ are parametrized by a Fano surface $F$. Choose a generic line $\ell$ in $X$ corresponding to a point in $F$, and let $\tilde{C}_{\ell}$ be the subvariety of lines in $X$ incident to $\ell$. The blowup of $X$ along $\ell$ has the structure of a conic bundle over $\mathbb{P}^{2}$ whose discriminant curve $C_{\ell}$ is a smooth plane quintic. The curve $\tilde{C}_{\ell}$ is an unramified double cover of $C_{\ell}$. The pair $\left(\tilde{C}_{\ell}, \iota\right)$ is a point in $\mathcal{R}_{6}$, and the Prym variety $P\left(\tilde{C}_{\ell}, \iota\right)$ is independent of $\ell$ ([M1], Section 1$)$. This construction of $\left(\tilde{C}_{\ell}, \iota\right)$ can be extended to cubic threefolds with nodes. Now we construct a singular plane quintic and a double cover for the Segre Cubic.

Definition 5. For any field of characteristic $\neq 2$ the Segre Cubic $\mathcal{S}$ in $\mathbb{P}^{4}$ is given by the equation

$$
\left\{\left(x_{0}, \ldots, x_{4}\right): \sum_{i \neq j \neq k} 2 x_{i} x_{j} x_{k}+\sum_{i \neq j} x_{i}^{2} x_{j}=0, \quad i, j, k \in\{0, \ldots, 4\}\right\} .
$$

One node is given by the coordinates $(1: 1: 1:-1:-1)$, and the other nine are obtained by permuting these coordinates.

We can obtain $\mathcal{S}$ as follows: Let $p_{1}, \ldots, p_{5}$ be points in general position in $\mathbb{P}^{3}$, and for each pair of points let $\ell_{i j}$ be the line joining $p_{i}$ and $p_{j}$. Blow up $\mathbb{P}^{3}$ at the five points, and then blow down the proper transforms of the ten lines $\ell_{i j}$. The image is $\mathcal{S}$ in $\mathbb{P}^{4}([\mathrm{H}], 3.1-3.2)$.

There are 15 planes contained in $\mathcal{S}$. Ten of them come from proper transforms of the planes in $\mathbb{P}^{3}$ containing three points $p_{i}, p_{j}$ and $p_{k}$. These are labeled $\Pi_{i j k}$ 
$(i<j<k)$. The other five are images of the exceptional divisors coming from the blowup. These are labeled $\Pi_{i}(1 \leq i \leq 5)$. Each of the 15 planes contains 4 nodes.

$\mathcal{S}$ contains at least 6 two-dimensional families of lines, $R_{i}(1 \leq i \leq 5)$, which are proper transforms of lines through $p_{i}$ and $R_{0}$, which is the family of twisted cubics through the five points. Each line in $R_{i}$ goes through the five planes $\Pi_{i j k}$ $(i<j<k)$. Each line in $R_{0}$ goes through $\Pi_{i}$ ([SR], VIII 2.32).

Choose a line $\ell$ in $R_{1}$ that does not go through a node and goes through exactly 5 planes, and project from it onto $\mathbb{P}^{2}$. The image of each of the five planes is a line in the degeneracy locus for $\mathcal{S}$. Each line meets each of the other four lines at a node. Each node is where the preimage is a plane containing a node on $\mathcal{S}$. Let $C_{0}$ be the plane quintic consisting of these 5 lines. We label each line $L_{i}, 1 \leq i \leq 5$. The dual graph of $C_{0}$ is the complete graph on five vertices $K_{5}$.

We now describe a double cover for the curve $C_{0}$ above. The preimage of each point in $C_{0}$ consists of two lines, excluding $\ell$, which are points in an étale double cover $\tilde{C}_{0}$ interchanged by the involution $\iota$. The whole cover can be described as follows: Start with ten copies of $\mathbb{P}^{1}, L_{i}^{\epsilon}$ where $1 \leq i \leq 5$ and $0 \leq \epsilon \leq 1$. Each line $L_{i}^{\epsilon}$ has four points marked on it, $p_{i, j}^{\epsilon}$ where $1 \leq \bar{j} \leq \overline{5}$ and $j \neq i$. In the following the notation $\left(p_{i, j}^{0} \sim p_{j, i}^{1}\right)$ means that the points $p_{i, j}^{0}$ and $p_{j, i}^{1}$ are identified. The double curve $\tilde{C}_{0}$ is

$$
\tilde{C}_{0}=\left(\coprod_{i, \epsilon} L_{i}^{\epsilon}\right) /\left(p_{i, j}^{0} \sim p_{j, i}^{1}\right) .
$$

The dual graph $\Gamma_{0}=\Gamma\left(\tilde{C_{0}}\right)$ appears in Figure 1. If the vertices of $\Gamma\left(C_{0}\right)$ are labeled $v_{j}$, then the vertices $a_{j}$ and $b_{j}$ both map to $v_{j}$. The map of edges is given by the map of vertices.

\section{Proof of the main theorem}

We now prove Theorem 1 First we give a degenerating family of cubic threefolds and the associated double covers of quintic curves. Let $\ell_{0}$ be a line in $\mathcal{S}$ (chosen as in Section 2). Choose a smooth cubic threefold $X$ such that it also contains $\ell_{0}$. We have a pencil of cubic threefolds that contain $\ell_{0}$ :

$$
X_{a, b}=a X+b \mathcal{S}, \quad(a: b) \in \mathbb{P}^{1} .
$$

By restricting to some open subset $S$ of $\mathbb{P}^{1}$ such that the only singular threefold is $\mathcal{S}$ over $0=(0: 1)$ we get a family $\mathcal{X} / S$ of cubic threefolds. Let $U=S-\{(0: 1)\}$. For each $s \in U$ there is a pair $\left(\tilde{C}_{s}, \iota\right)$. The family of such pairs is a flat family over $U$. By direct calculation the limit is $\left(\tilde{C}_{0}, \iota\right)$.

Lemma 6. Let $\left(\tilde{C}_{0}, \iota\right)$ be as above. Then the map $\overline{\mathcal{R}_{6}} \rightarrow \overline{\mathcal{A}_{5}^{\text {vor }}}$ is regular in the neighborhood of $\left(\tilde{C}_{0}, \iota\right)$.

Proof. Here we will use Lemma 4. Let $\Gamma_{0}$ be as above. Suppose $\Gamma_{1}$ and $\Gamma_{2}$ are subgraphs with $\iota\left(\Gamma_{i}\right)=\Gamma_{i}(i=1,2)$. Then the first possible case is that $\Gamma_{1}$ has 2 vertices and $\Gamma_{2}$ has 8 vertices. If the vertices in $\Gamma_{1}$ correspond to the lines $L_{1}^{0}$ and $L_{1}^{1}$ (using the notation from Section 2), then the lines $L_{1}^{0}$ and $L_{1}^{1}$ do not meet. So on the dual graph their corresponding vertices do not have an edge between them. Then $\Gamma_{1}$ is not connected and so the conditions of Lemma 4 are satisfied. The second possible case is if $\Gamma_{1}$ and $\Gamma_{2}$ have 4 and 6 vertices, respectively. Suppose 


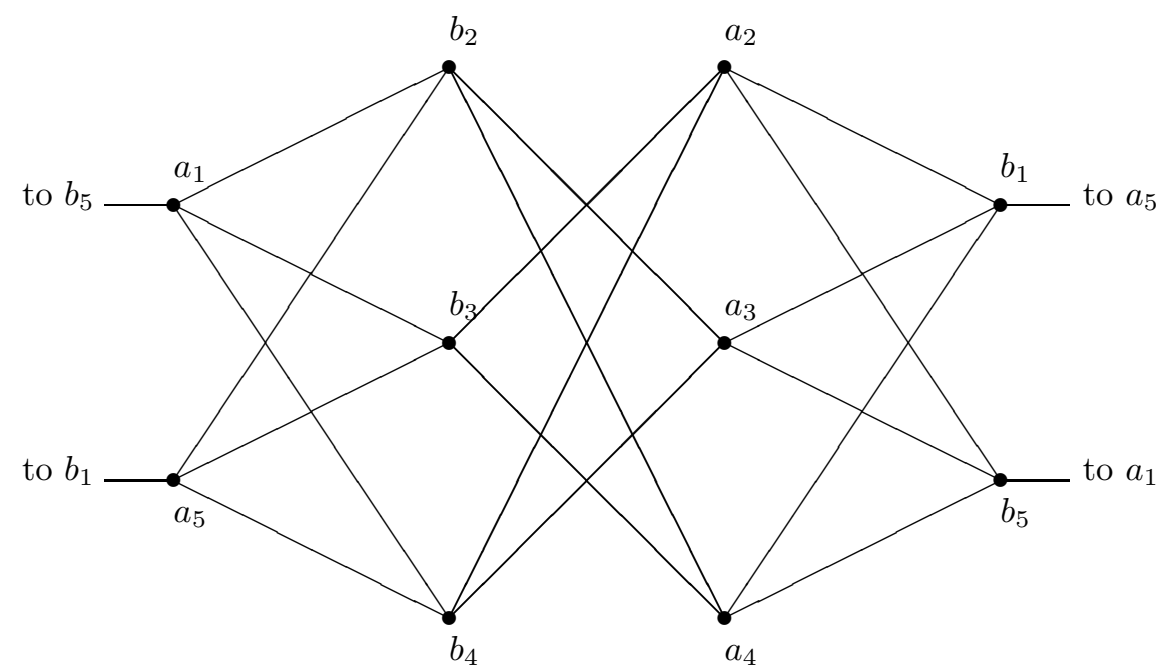

Figure 1 . The edges are named as follows: $e_{1}=\left(b_{3}, a_{2}\right), e_{2}=$ $\left(a_{4}, b_{2}\right), e_{3}=\left(a_{5}, b_{3}\right), e_{4}=\left(a_{5}, b_{4}\right), e_{5}=\left(a_{5}, b_{1}\right), e_{6}=\left(a_{4}, b_{3}\right)$, $e_{7}=\left(b_{3}, a_{1}\right), e_{8}=\left(b_{2}, a_{5}\right), e_{9}=\left(a_{1}, b_{4}\right), e_{10}=\left(b_{2}, a_{1}\right)$. The rest of the edges are named as follows: if edge $e_{i}$ is $\left(a_{j}, b_{k}\right)\left(\left(b_{j}, a_{k}\right)\right.$ respectively) the edge $e_{i}^{\prime}$ is $\left(b_{j}, a_{k}\right),\left(\left(a_{j}, b_{k}\right)\right.$ respectively).

The tree used to form the basis of $H_{1}(\Gamma, \mathbb{Z})$ is given by the edges $e_{6}=\left(a_{4}, b_{3}\right), e_{7}=\left(b_{3}, a_{1}\right), e_{8}=\left(b_{2}, a_{5}\right), e_{9}=\left(a_{1}, b_{4}\right), e_{10}=$ $\left(b_{2}, a_{1}\right)$ and $e_{10}^{\prime}=\left(a_{2}, b_{1}\right), e_{7}^{\prime}\left(a_{3}, b_{1}\right), e_{9}^{\prime}=\left(b_{1}, a_{4}\right), e_{8}^{\prime}=\left(a_{2}, b_{5}\right)$.

without loss of generality that the lines $L_{1}^{0}, L_{1}^{1}, L_{2}^{0}$ and $L_{2}^{1}$ are in $\Gamma_{1}$. This would imply that $\Gamma_{1}$ is not connected, because the connected subgraph with $L_{1}^{0}$ and $L_{2}^{1}$ is not connected with the subgraph with $L_{1}^{1}$ and $L_{2}^{0}$. Therefore the map is regular around $\mathcal{S}$.

It follows that the associated family of Prym varieties has the compactified Prym variety of $\left(\tilde{C}_{0}, \iota\right)$ as its limit over 0 .

Let us now compute the corresponding dicing. By the above lemma, we only need to compute $X^{-} \subset C_{1}(\Gamma, \mathbb{Z})$ as in Definition 3 and show it is $R_{10}$. The following is a basis for $H_{1}(\Gamma, \mathbb{Z})$ :

$$
\begin{aligned}
h_{1}=e_{6}^{\prime}+e_{7}^{\prime}-e_{9}^{\prime}+e_{6}+e_{7}-e_{9}, & h_{7}=e_{5}-e_{9}^{\prime}+e_{6}+e_{7}+e_{10}+e_{8}, \\
h_{2}=e_{1}^{\prime}+e_{7}^{\prime}+e_{9}^{\prime}+e_{6}+e_{7}+e_{10}, & h_{8}=e_{4}+e_{9}+e_{10}-e_{8}, \\
h_{3}=e_{1}-e_{10}^{\prime}-e_{9}^{\prime}+e_{6}, & h_{9}=e_{3}+e_{7}+e_{10}-e_{8}, \\
h_{4}=e_{2}+e_{6}+e_{7}+e_{10}, & h_{8}^{\prime}=e_{4}^{\prime}+e_{9}^{\prime}+e_{10}^{\prime}-e_{8}^{\prime}, \\
h_{5}=e_{2}^{\prime}-e_{10}^{\prime}-e_{9}^{\prime}+e_{6}+e_{7}-e_{9}, & h_{9}^{\prime}=e_{3}^{\prime}+e_{7}^{\prime}+e_{10}^{\prime}-e_{8}^{\prime} . \\
h_{6}=e_{5}^{\prime}-e_{8}^{\prime}-e_{10}^{\prime}-e_{9}^{\prime}+e_{6}+e_{7}, &
\end{aligned}
$$


The basis for $X^{-}$is as follows:

$$
\begin{aligned}
& \ell_{1}=\frac{1}{2}\left(h_{2}-\iota\left(h_{2}\right)\right)=\frac{1}{2}\left(h_{3}-\iota\left(h_{3}\right)\right), \\
& \ell_{2}=\frac{1}{2}\left(h_{4}-\iota\left(h_{4}\right)\right)=\frac{1}{2}\left(h_{5}-\iota\left(h_{5}\right)\right), \\
& \ell_{3}=\frac{1}{2}\left(h_{9}-\iota\left(h_{9}\right)\right)=\frac{1}{2}\left(h_{9}^{\prime}-\iota\left(h_{9}^{\prime}\right)\right), \\
& \ell_{4}=\frac{1}{2}\left(h_{8}-\iota\left(h_{8}\right)\right)=\frac{1}{2}\left(h_{8}^{\prime}-\iota\left(h_{8}^{\prime}\right)\right), \\
& \ell_{5}=\frac{1}{2}\left(h_{6}-\iota\left(h_{6}\right)\right)=\frac{1}{2}\left(h_{7}-\iota\left(h_{7}\right)\right),
\end{aligned}
$$

and $X^{-}=\left\langle\ell_{1}, \ldots, \ell_{5}\right\rangle$. The dicing is obtained by seeing how the edges $e_{j}$ restrict to $X^{-}$. The matrix for the dicing of $X^{-} \otimes \mathbb{R}$ is $\left(a_{i j}\right)$ where $a_{i j}$ is defined to be 1 if $\ell_{i}$ contains $e_{j}$. The matrix we get is

$$
\left(\begin{array}{lllll|lllll}
1 & 0 & 0 & 0 & 0 & 1 & 0 & 0 & 1 & 1 \\
0 & 1 & 0 & 0 & 0 & 1 & 1 & 0 & 0 & 1 \\
0 & 0 & 1 & 0 & 0 & 0 & 1 & 1 & 0 & 1 \\
0 & 0 & 0 & 1 & 0 & 0 & 0 & 1 & 1 & 1 \\
0 & 0 & 0 & 0 & 1 & 1 & 1 & 1 & 1 & 1
\end{array}\right),
$$

which is $R_{10}$. This concludes the proof of Theorem 1

\section{Applichtion to Cubic threefolds}

As a corollary of Theorem 1 we get Theorem 2 The theorem we will use in the proof is

Theorem 7 ([M2], Thm. 3.11). Let $\operatorname{char}(k) \neq 2$. Let $X$ be a nonsingular cubic threefold in $\mathbb{P}^{4}$, defined over $k$. If there exists a birational transformation between $X$ and $\mathbb{P}^{3}$, then the canonically polarized Prym variety $(P(X), \Xi)$ associated with $X$ is isomorphic, as a polarized abelian variety, to a product of canonically polarized Jacobian varieties of curves.

The proof of Theorem 2 now follows easily. If the threefolds in the family from section 3 were rational, then by Theorem [7the family of degenerating Pryms would be a family of degenerating Jacobians or product of Jacobians. Recall from section 1 that a degenerating family of curves would give a cographic unimodular system. But by Theorem 1 we get $R_{10}$, which is not cographic. This gives a contradiction.

Generic, as used in Theorem 2, means that in every one-parameter family of cubic threefolds degenerating to the Segre Cubic as in the proof of Theorem 1 there are only finitely many smooth rational cubic threefolds. Using the fact that the locus of products of canonically polarized Jacobians of curves in $\mathcal{A}_{5}$ is closed, it can be shown that the smooth rational threefolds are contained in a Zariski proper closed subset of the parameter space of smooth cubic threefolds.

In previous proofs of Theorem 2 which used degenerations, the limiting (generalized) Prym was an extension of a nontrivial Abelian variety by a torus. In $[\mathrm{C}]$ the Prym is an extension by a torus $k^{*}$, and in $\left[\mathrm{B}\right.$ ] the torus is $\left(\mathbb{C}^{*}\right)^{2}$. In this proof the extension is the maximal possible, as shown in the following proposition.

Proposition 8. The generalized Prym associated to the double cover $\left(\tilde{C}_{0}, \iota\right)$ is $\left(k^{*}\right)^{5}$. 
Proof. This follows from the fact that the generalized Prym is an extension of an Abelian variety by a torus ( $[\mathrm{ABH}]$, Section 1$)$. The dimension of the torus is given by $h_{1}\left(\tilde{C}_{0}, \mathbb{Z}\right)-h_{1}\left(C_{0}, \mathbb{Z}\right)=5$. The abelian part must therefore be 0 .

\section{ACKNOWLEDGEMENTS}

I would like to thank Prof. Valery Alexeev for his invaluable help and attention to my work and Prof. Bernd Sturmfels for his help with Macaulay2. I would also like to thank Prof. Roy Smith and Prof. Robert Varley for their help and suggestions in the course of preparing this paper. The project was partially supported by NSF grant 0101280 .

\section{REFERENCES}

$[\mathrm{ABH}]$ V. Alexeev, Ch. Birkenhake and K. Hulek, Degenerations of Prym Varieties, J. Reine Angew. Math. 553(2002), 73-116. MR1944808 (2003k:14033)

[B] F. Bardelli, Polarized Mixed Hodge Structures: on Irrationality of Threefolds via Degeneration, Ann. Mat. Pura Appl. (4), 137 (1984), 287-369. MR0772264 (86m:14030)

[C] A. Collino, A Cheap Proof of the Irrationality of Most Cubic Threefolds. Boll. Un. Mat. Ital. (5), 16-B (1979), 451-465. MR0546468 (80i:14011)

[CG] C. Herbert Clemens and Phillip A. Griffiths, The Intermediate Jacobian of the Cubic Threefold, Annals of Mathematics, 95 (1972), 281-356. MF 0302652 (46:1796)

[DG] V. Danilov and V. Grishukhin, Maximal Unimodular Systems of Vectors, Europ. J. Combinatorics, 20 (1999), 507-526. MR1703596 (2000d:15004)

[H] B. Hunt, The Geometry of Some Special Arithmetic Quotients, Lecture Notes in Mathematics, vol. 1637, Springer-Verlag 1996. MR1438547 (98c:14033)

[M1] J. P. Murre, Algebraic Equivalence Modulo Rational Equivalence on a Cubic Threefold, Compositio Mathematica, 25 (1972), Fasc. 2, 161-206. MR0352088|(50:4576a)

[M2] J. P. Murre, Reduction of the Proof of the Non-Rationality of a Nonsingular Cubic Threefold to a Result of Mumford, Compositio Mathematica, 27 (1973), Fasc. 1, 63-82. MR 0352089 (50:4576b)

[O] J. G. Oxley, Matroid Theory, Oxford University Press, 1992. MR1207587 (94d:05033)

[SR] J. G. Semple and L. Roth, Introduction to Algebraic Geometry, Clarendon Press, Oxford, 1985. MR0034048 (11:535d)

[V] V. Vologodsky, Locus of Indeterminacy of the Prym Map, J. Reine Angew. Math. 553(2002), 117-124. MF1944809 (2003i:14036)

Department of Mathematics, University of Georgia, Athens, Georgia 30602 